\title{
AC 2008-2219: EXPERIMENTALLY DETERMINING MASS MOMENT PROPERTIES
}

\section{Byron Newberry, Oklahoma Christian University of Science and Arts}

Dr. Byron Newberry is Associate Professor and Chair of Mechanical Engineering at Oklahoma Christian University. He holds a B.S. degree in Mechanical Engineering from Oklahoma Christian University and M.S. and Ph.D. degrees in Mechanical Engineering from The University of Michigan, Ann Arbor. His interests include stress analysis, nonlinear dynamics, structural vibration, and engineering design. 


\section{Experimentally Determining Mass Moment Properties}

\section{Abstract}

A project is presented that requires students to experimentally determine the mass moment properties for a three-dimensional test specimen. The project begins by attaching the test specimen (a composite structure made from small wooden blocks) to a pendulum platform of known mass properties. The platform is equipped with an accelerometer to allow dynamic motion tracking. Multiple swing tests are performed and the dynamic response of the loaded pendulum is recorded using a National Instruments data acquisition system. Using an oscillator model and Fourier analysis, the students calculate both the damping ratio and the damped natural frequency of the loaded platform. Armed with these results and the known pendulum geometry, the students back calculate the mass moment properties of the test specimen. The students also estimate the inertia properties using composite body methods and CAD software to allow comparison of the experimental results. An error analysis of discrepancies between the three methods is required. The project has been found to effectively demonstrate the significance of mass properties in determining the dynamics of a system. Furthermore, the project is a challenging experimental assignment that requires mature experimental techniques; as the results are highly sensitive to inaccuracies in measuring the dynamic response.

Introduction and Motivation

A theory course in dynamics is typically included at the sophomore level of most mechanical engineering programs ${ }^{1,2}$. It is common for the content of such a course to begin with particle dynamics. For such analyses the student need only know the mass of the particle(s) involved; usually given in the problem. The course content progresses to rigid body dynamics. With the introduction of rigid bodies, an understanding of the distribution of the mass within the body is required. Typically, however, that said distribution is again given or is readily determined. As a result, students tend to view the inertial properties of a body as a trivial input to a mathematical problem; similar to the length of a connector or the mass of a component. In reality, determining the inertia properties of a real structure can be quite challenging.

The project presented herein demonstrates the link between the inertia properties and the dynamic response of the rigid body; in as much as the dynamic response can actually be used to determine unknown inertia. The project is part of a junior-level course entitled Experimental Mechanics taught at Oklahoma Christian University. The course focuses on experimental investigations directly related to the theory learned in Strength of Materials and Dynamics (both prerequisites to this course).

\section{Project Overview}

The students are assigned the simple objective of determining the inertia properties of a rigid body analytically, computationally, and experimentally. Figure 1 shows a typical rigid body sample used within the course. Each sample is fabricated from wooden building blocks and no two samples are identically configured. 

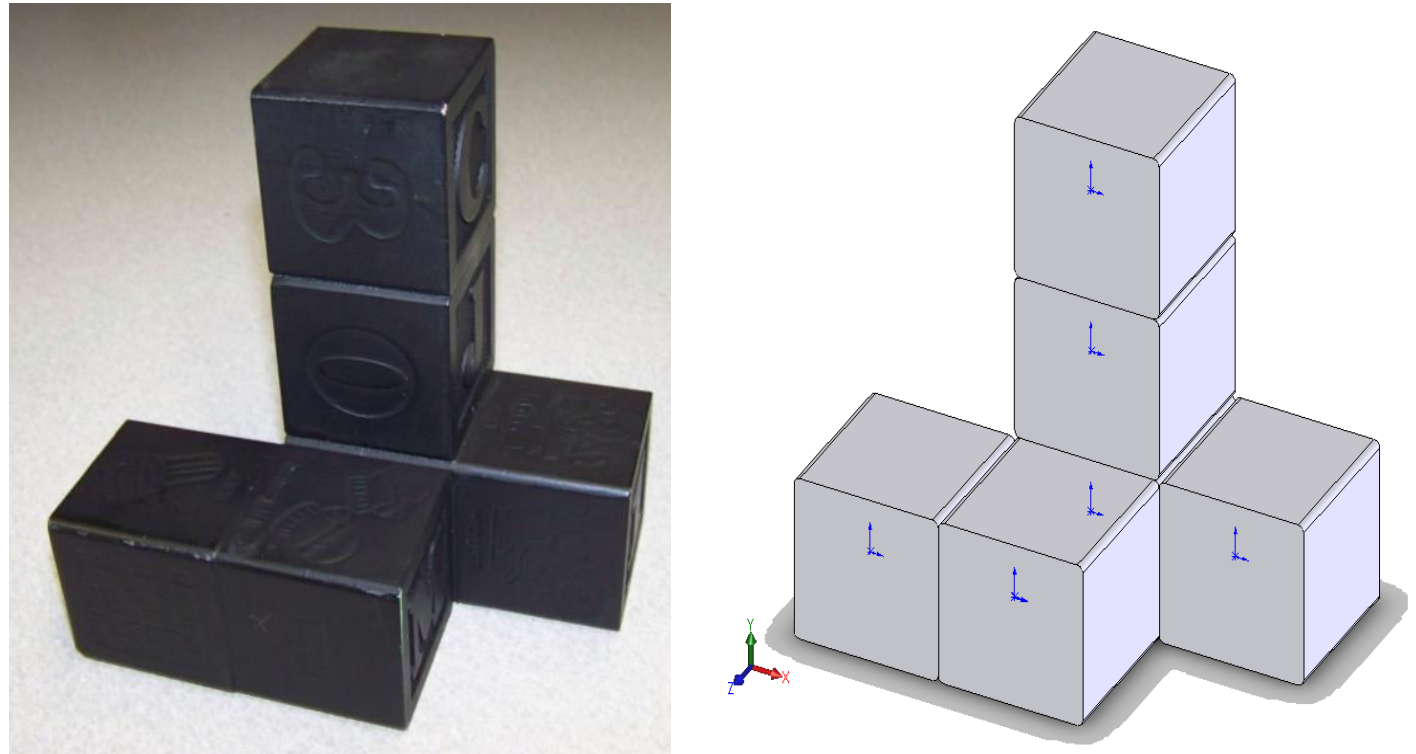

Figure 1 - Actual rigid body sample and its corresponding solid body model

The students begin by using analytical methods to determine $\mathrm{I}_{\mathrm{xx}}, \mathrm{I}_{\mathrm{yy}}$, and $\mathrm{I}_{\mathrm{zz}}$ about the mass center of the sample. This requires first estimating the density of the sample using measured mass and volume data. Once completed, the students proceed to generate a 3D solid model of the specimen. Solidworks is specifically used, though any 3D modeling packages would suffice ${ }^{3}$. The complete inertia tensor for the specimen is directly provided by the correctly generated 3D model.

These analytical and computation results form a comparison standard for the remaining experimental work. Note that both of these models have made two unverified assumptions.

1. It is assumed that all the blocks used within the sample are of identical material and that the material is isotropic. This assumption is almost certainly false given the wood from which the blocks are fabricated.

2. The students are told nothing of how the blocks are connected together and they are not allowed to destructively examine the sample. Students will assume a simple glue bond with negligible mass.

These assumptions are representative of the complexity present when attempting to determine the inertia properties of more complex rigid bodies using analytical and/or computational methods. Accounting for fabrication methods (bolts, welds, etc.), material variations, and complex hidden geometries becomes very challenging in all but the most simple of cases.

\section{Theory Overview}

Figure 2 shows a schematic of a loaded pendulum. The upper circle (marked P) represents the mass center of the pendulum located a distance $\mathrm{L}_{\mathrm{p}}$ from the pivot. The pendulum has rotational inertia 
about the center of mass of $I_{p}$. The rotational inertia of the pendulum about the pivot is $I_{p}+m_{p} L_{p}^{2}$. A mass sample is placed on the pendulum (the lower circle marked $\mathrm{S}$ ). The sample has mass $\mathrm{m}_{\mathrm{s}}$ and local inertia about the mass center of $I_{s}$. The center of mass of the sample is located a distance $\mathrm{L}_{\mathrm{s}}$ from the pivot of the pendulum.

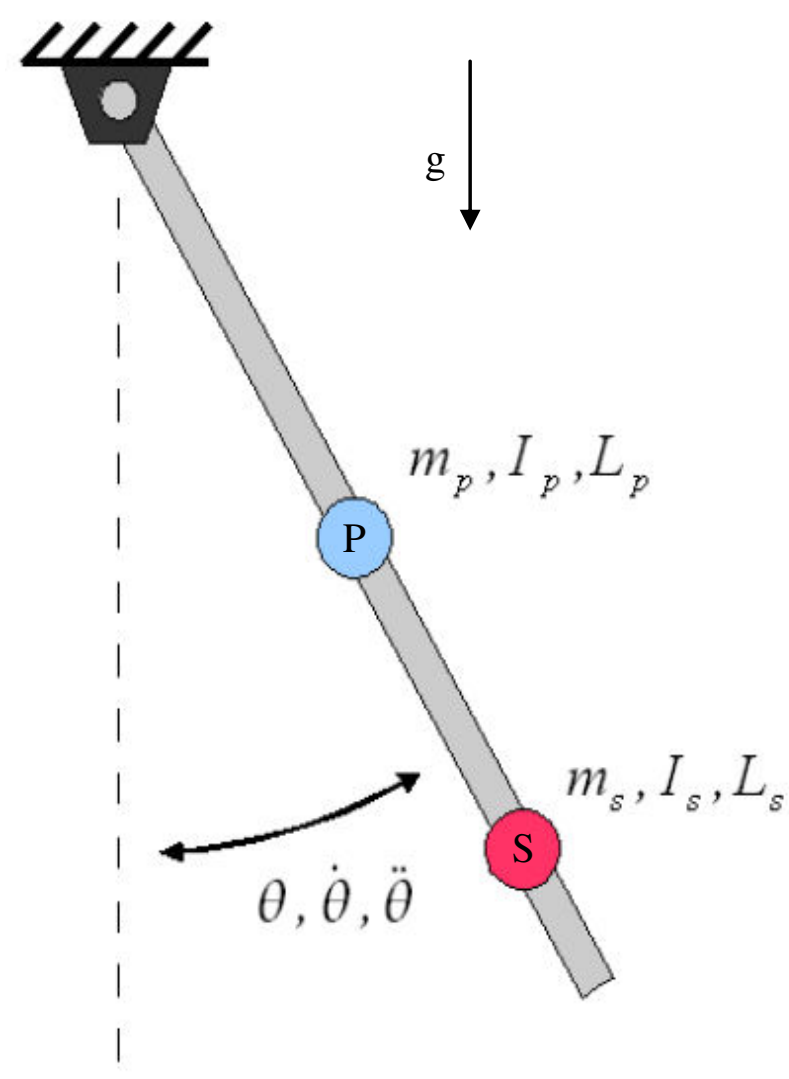

Figure 2 - Pendulum schematic diagram

The entire pendulum oscillates, characterized by the variable $\theta$ and its derivatives. The governing equation for the system may be stated (small angles being assumed) as follows.

$$
\ddot{\theta}+\left[\frac{g\left(m_{p} L_{p}+m_{s} L_{s}\right)}{\left(I_{p}+m_{p} L_{p}^{2}\right)+\left(I_{s}+m_{s} L_{s}^{2}\right)}\right] \theta=0
$$

Rewriting this expression in standard form allows definition of the natural frequency of the loaded pendulum.

$$
\ddot{\theta}+\omega_{n}^{2} \theta=0 \quad \text { where } \omega_{n}=\sqrt{\frac{g\left(m_{p} L_{p}+m_{s} L_{s}\right)}{\left(I_{p}+m_{p} L_{p}^{2}\right)+\left(I_{s}+m_{s} L_{s}^{2}\right)}}
$$


The mass moment of the pendulum and the sample may be determined as shown; with $\omega_{\mathrm{np}}$ denoting the undamped frequency of the pendulum and $\omega_{\text {ns }}$ the undamped frequency of the pendulum with a sample attached.

$$
I_{p}=\frac{g\left(m_{p} L_{p}\right)}{\omega_{n p}{ }^{2}}-m_{p} L_{p}^{2} \quad \text { and } \quad I_{s}=\frac{g\left(m_{p} L_{p}+m_{s} L_{s}\right)}{\omega_{n s}{ }^{2}}-\left(I_{p}+m_{p} L_{p}^{2}\right)-m_{s} L_{s}^{2}
$$

To account for energy dissipation, which is present in the experimental model, damping proportional to the angular velocity may be adopted. The governing expression becomes the following.

$$
\ddot{\theta}+2 \zeta \omega_{n} \dot{\theta}+\omega_{n}^{2} \theta=0 \quad \text { with } \quad \omega_{d}=\sqrt{1-\zeta^{2}} \omega_{n}
$$

The damping ratio $\zeta$ is initially unknown. It may be experimentally determined using the decay envelop of the measured pendulum oscillations and a logarithmic decrement analysis ${ }^{4}$. In practice, the damping ratio will result in a very small correction as the damping ratio is typically much less that $5 \%$ for the experimental setup presented.

\section{Experimental Implementation}

The experimental setup is shown in Figure 3. The plastic pendulum has been generated using a Dimension SST 768 Series 3D printer ${ }^{5}$. Though not absolutely required, use of the 3D printer affords the luxury of generating a strong, yet hollow, pendulum. The results of the project improve when the mass of the pendulum remains small so as to not dominate the inertia of the overall system. Another key feature of the pendulum is the location of the sample platform. If the distance between the axis of rotation and the mass center of the sample $\left(\mathrm{L}_{\mathrm{s}}\right)$ becomes too large, the parallel axis correction dominates the system inertia (i.e. the local inertia about the mass center becomes negligible).
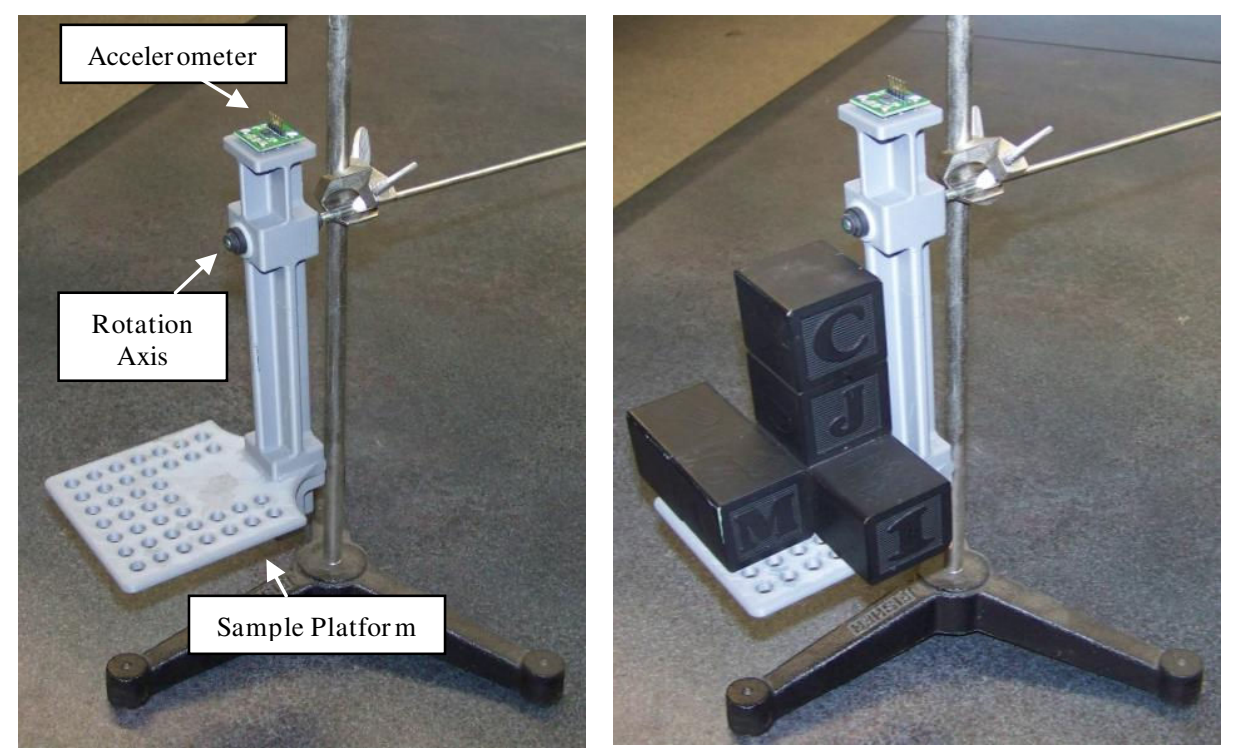

Figure 3 - Experimental pendulum configuration (without and with sample) 
Located at the top of the pendulum is an Analog Devices ADXL320 accelerometer that has been configured to output a voltage proportional to the component of gravity perpendicular to the pendulum $\operatorname{arm}^{6}$. When the pendulum is vertical (rest position), the output of the accelerometer (appropriately conditioned) is zero volts. As the pendulum oscillates, the output signal from the accelerometer becomes either negative or positive; depending on the tilt angle of pendulum.

Figure 4 presents the experimental process required to successfully complete the project. Note that the pendulum is first tested without a sample (the yellow boxes). The inertia properties of the pendulum are needed to allow calculation of the sample's mass moment of inertia. If desired, the mass moment properties of the pendulum could be provided to the students (eliminating the steps shown in yellow below).

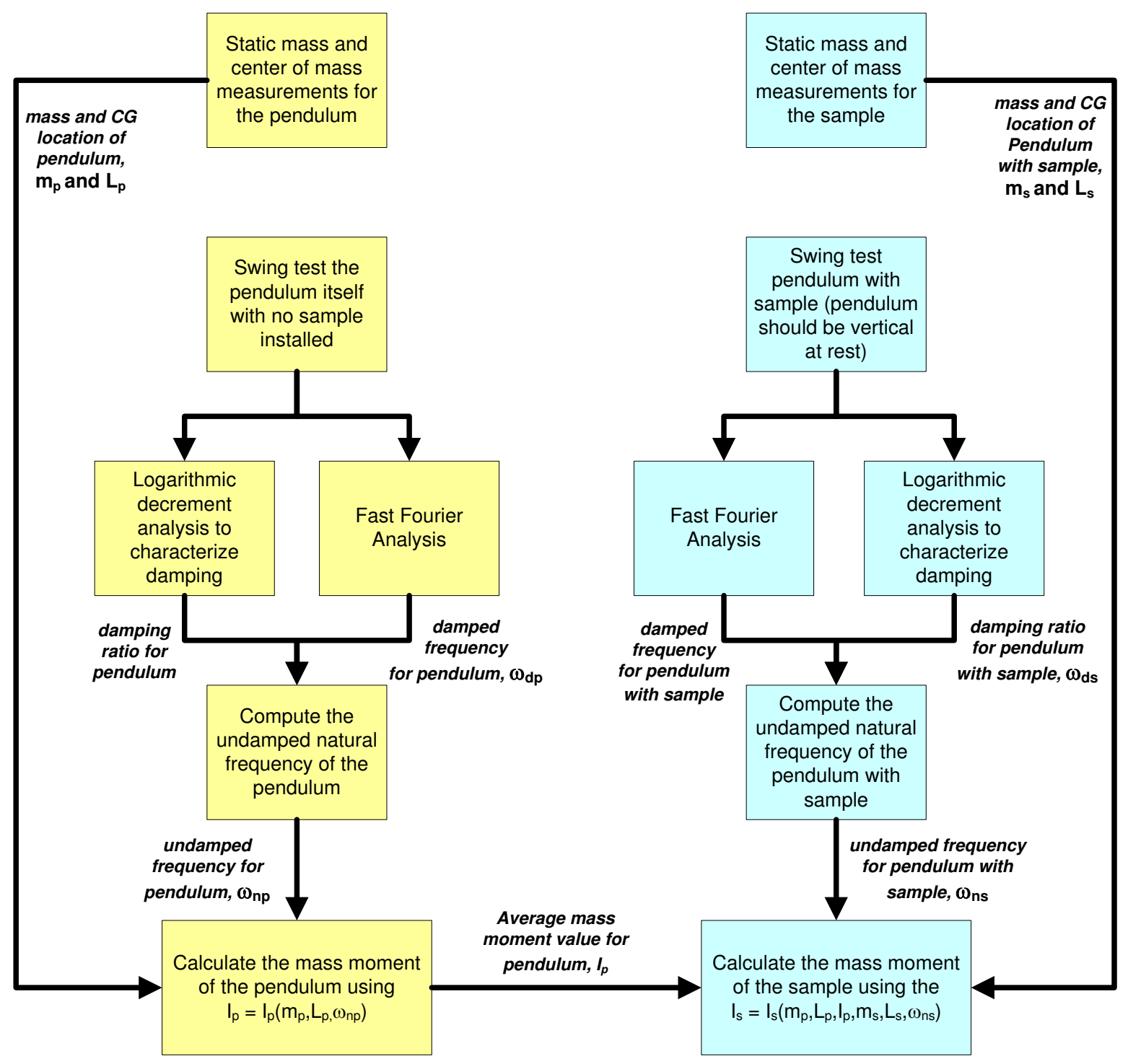

Figure 4 - Flowchart of the experimental procedure (yellow steps address the pendulum only, blue steps include the sample). Repeat for each axis of the sample. 
It is necessary to collect a relatively large amount of data to perform the Fourier analysis used in determining the natural frequency of the dynamic pendulum. Labview, in combination with a National Instruments NI USB-6009 data acquisition module, is used to collect the voltage output of the accelerometer at a sample rate of 500 times/second as the pendulum oscillates ${ }^{7}$.

It should be noted that the students taking this course have already received exposure to Fourier analysis, but typically have yet to use a Fast Fourier Transform (FFT). This project provides an excellent opportunity to introduce the theory of the FFT and to discuss its computational implementation in modern software packages. Mathcad and/or Matlab are specifically used, both offering implementations of the FFT that require minimal user programming ${ }^{8,9}$.

\section{Project Educational Strengths}

When the project is assigned, students anticipate few difficulties given what seems a systematic experimental procedure. This response is consistent with the reality of textbook problems. Students naively believe that it should be simple to solve for any unknown in an equation; requiring only a bit of algebra. Thus, given the natural frequency of the dynamic response, one should be able to back-solve for the inertia. It is common, however, for students to find the experimental inertia results differ by as much as $30 \%$ when compared to the analytical and computational results.

The reality is that back-solving for inertia is highly sensitive to experimental inaccuracies in the measured natural frequency. Figure 5 shows a typical dynamic response for the pendulum (without the sample). Note the width of the frequency response peak. It is very difficult to precisely know the frequency of the dynamic response.
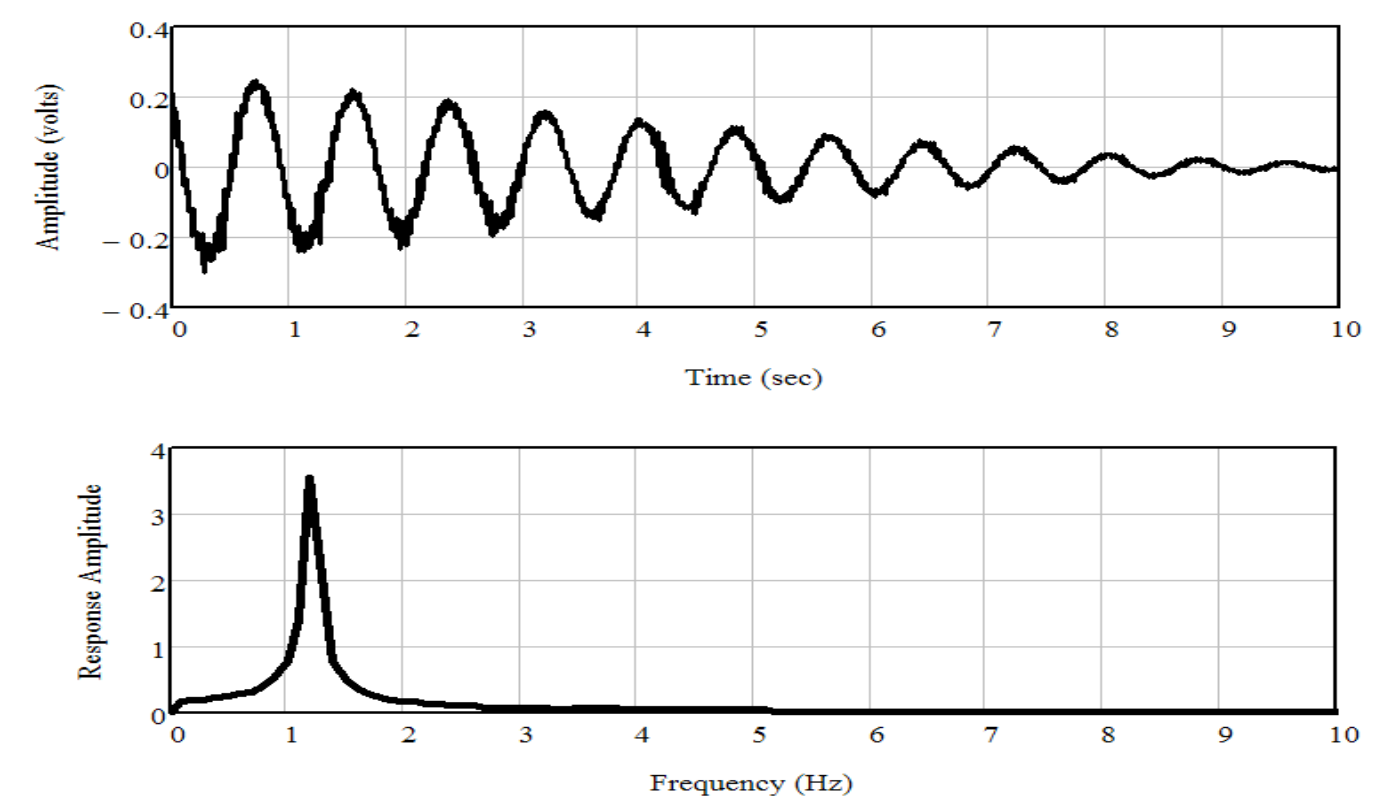

Figure 5 - Dynamic response for unloaded pendulum (500 samples/sec) 
In Figure 6, typical experimental results are tabulated. The standard deviation in the measured frequency is approximately $3.3 \%$. When the response frequency is used to compute the mass moment of the pendulum, however, the standard deviation in the results is magnified to $22.7 \%$. Likewise, the mean mass moment estimate for the sample is found to be $2.18 \mathrm{lb}-\mathrm{in}^{2}$ with a standard deviation of $23.2 \%$. Yet, while this standard deviation is large, the mean experimental mass moment value favorably compares to the computational estimate of $2.02 \mathrm{lb}-\mathrm{in}^{2}$ for the sample in question (a difference of $7.6 \%$ ).

\begin{tabular}{|c|c|c|c|c|c|c|c|c|}
\hline & \multicolumn{4}{|c|}{ Measured Response Frequency $(\mathrm{Hz})$} & \multicolumn{4}{|c|}{ Mass Moment Results (lb-in $\left.{ }^{2}\right)$} \\
\hline & Max & Min & Avg. & Std.Dev. & Max & Min & Avg. & Std.Dev. \\
\hline Pendulum & 1.30 & 1.18 & 1.21 & $3.3 \%$ & 4.82 & 1.96 & 3.29 & $22.7 \%$ \\
\hline Pendulum with sample & 1.39 & 1.33 & 1.36 & $1.9 \%$ & & & & \\
\hline Sample (mean pendulum results) & & & & & 2.87 & 1.61 & 2.18 & $23.2 \%$ \\
\hline
\end{tabular}

Figure 6 - Example experimental results for a single axis of one sample. The mean results for the pendulum were used when computing the mass moment results for the sample.

Faced with the need to precisely determine the natural frequency of oscillation, the class as a whole is required to examine the experimental procedure and propose means of improving the results. A typical proposal is to incorporate a damping correction within the frequency calculation (students generally begin by assuming $\omega_{d}=\omega_{n}$ ). A logarithmic decrement analysis of the decay envelop will reveal this correct is very slight.

In fact, however, damping is a barrier to accurately estimating the frequency response. The granularity of the FFT is linked to the length of the dynamic response time window recorded. A longer time window affords greater accuracy. The decay rate of the dynamic response prevents recording a longer time window, however. Referring to Figure 5, note the oscillations cease after approximately 10 cycles. Efforts are underway to produce a second generation pendulum with reduced friction losses at the pivot.

\section{Project Assessment}

At the time of this writing, this project has been used only twice. Given relatively small class sizes (10 to 14 students) and this limited number of offerings, quantitative outcome assessment results are currently not available. Student feedback, however, qualitatively reveals a strong appreciation for the integration of analytical, computational, and experimental analysis within a single experience. The investigative role necessary to reconcile the results from the various analysis methods creates a learning environment rarely if ever experienced within a theory course. The upper-level students taking this course consistently indicate an increased understanding of the link between dynamic response and the inertia of a system from having performed the project. Finally, the experimental nature of the project greatly increases student interest and motivation. This conclusion is not unexpected. The recent reports of Self, Borchert, and Redfield clearly demonstrate the positive influence that hands-on projects have on student motivation for learning $^{10,11}$. 


\section{Conclusions}

A hands-on approach to investigating the inertia properties of a rigid body has been presented. The project incorporates analytical, computational, and experimental analyses; requiring the students to conduct a critical comparison of the various results. The nature of the project presents experimental challenges that motivate maturing of the students understanding of the fundamental dynamics involved. Sufficient detail is presented to allow implementation within a typical mechanical engineering program.

\section{Bibliography}

1. Hibbeler, R.C., Engineering Mechanics - Dynamics, $10^{\text {th }}$ Edition, Pearson Prentice Hall, 2004.

2. Bedford, A. and Fowler, W., Engineering Mechanics - Dynamics, $4^{\text {th }}$ Edition, Pearson Prentice Hall, 2005.

3. SolidWorks 2007, www.solidworks.com.

4. James, M.L., Smith, G.M., Wolford, J.C., and Whaley, P.W., Vibration of Mechanical and Structural Systems, Harper \& Row Publishers, 1989.

5. Dimension 3D Printing Group, www.dimensionprint.com.

6. Analog Devices, www.analogdevices.com.

7. National Instruments, www.ni.com.

8. Mathcad 14, Parametric Technology Corporation (PTC), www.ptc.com.

9. Matlab 2007, The Mathworks, www.mathworks.com.

10. Self, B. and Redfield, R., "New Approaches in Teaching Undergraduate Dynamics", Proceedings of the 2001 ASEE Annual Conference \& Exposition, 2001.

11. Self, B., Borchert, R., and Redfield, R., "Evolution of an Introductory Dynamics Course through Continuous Assessment", Proceedings of the 2003 ASEE Annual Conference \& Exposition, 2003. 